\title{
Status of the Michigan Ultra-Cold Spin-Polarized Hydrogen Jet ${ }^{\text {a }}$
}

\author{
K. Yonehara*, B.K. Harris*, M.C. Kandes*, B.H. Kienman*, A.D. Krisch*, \\ M.A. Leonova*, V.G. Luppov*, V.S. Morozov*, J.B. Olson*, C.C. Peters*, \\ R.S. Raymond ${ }^{*}$, D.L. Sisco ${ }^{*}$, N.S. Borisov ${ }^{\dagger}$, V.V. Fimushkin, ${ }^{\dagger}$ and \\ A.F. Prudkoglyad**
}

*Spin Physics Center, University of Michigan, Ann Arbor, MI 48109-1120, USA

${ }^{\dagger} J$ oint Institute for Nuclear Research, RU-141980, Dubna, Russia

${ }^{* *}$ Institute for High Energy Physics, RU-142284, Protvino, Russia

\begin{abstract}
Progress on the Michigan ultra-cold proton-spin-polarized atomic-hydrogen Jet target is presented. We describe the present status of the Jet and some beam test results.
\end{abstract}

We are developing an ultra-cold high-density Jet target of proton-spin-polarized hydrogen atoms (Michigan Jet) to study spin effects in high energy collisions. The Jet uses a very high magnetic field and an ultra-cold separation cell coated with a superfluid ${ }^{4} \mathrm{He}$ film to produce a slow monochromatic electron-spin-polarized atomic hydrogen beam. This beam is focused by a parabolic mirror coated with superfluid ${ }^{4} \mathrm{He}$ and a superconducting sextupole magnet. An of transition unit will then convert this beam into a fully proton-spin-polarized beam [1].

A layout of the Michigan Jet is shown in Fig. 1. Atomic hydrogen is produced with a room-temperature if dissociator and guided to the ultra-cold $(170 \mathrm{mK})$ separation cell, which is coated with superfluid ${ }^{4} \mathrm{He}$ to suppress the surface recombination of hydrogen atoms, as shown in Fig. 2. The double walls of the cell form the mixing chamber of a dilution refrigerator. The cell's entrance and exit apertures are respectively located at about $95 \%$ and $50 \%$ of the superconducting solenoid's 12 Tesla magnetic field. After the hydrogen atoms are thermalized by collisions with the cell surface, the magnetic field gradient physically separates the atoms according to their electron-spin states. The "high-field-seeker" atoms in the two lowest hyperfine states $(|3\rangle$ and $|4\rangle)$ are attracted up toward the high field region and most escape from the cell; they quickly recombine on bare surfaces and are cryopumped away. The "low-field-seeker" atoms in the two higher hyperfine states $(|1\rangle$ and $|2\rangle)$ are accelerated toward the low field region and effuse from the exit aperture, forming a rather monochromatic electron-spin-polarized beam.

To increase the Jet density, we use a gold-plated copper focusing mirror with a polished surface covered with a ${ }^{4} \mathrm{He}$ superfluid film, which is similar to the prototype mirror [2]. After an rf transition unit [3], which will change state $|2\rangle$ atoms into state $|4\rangle$

\footnotetext{
a Supported by a research grant from the U.S. Department of Energy
} 


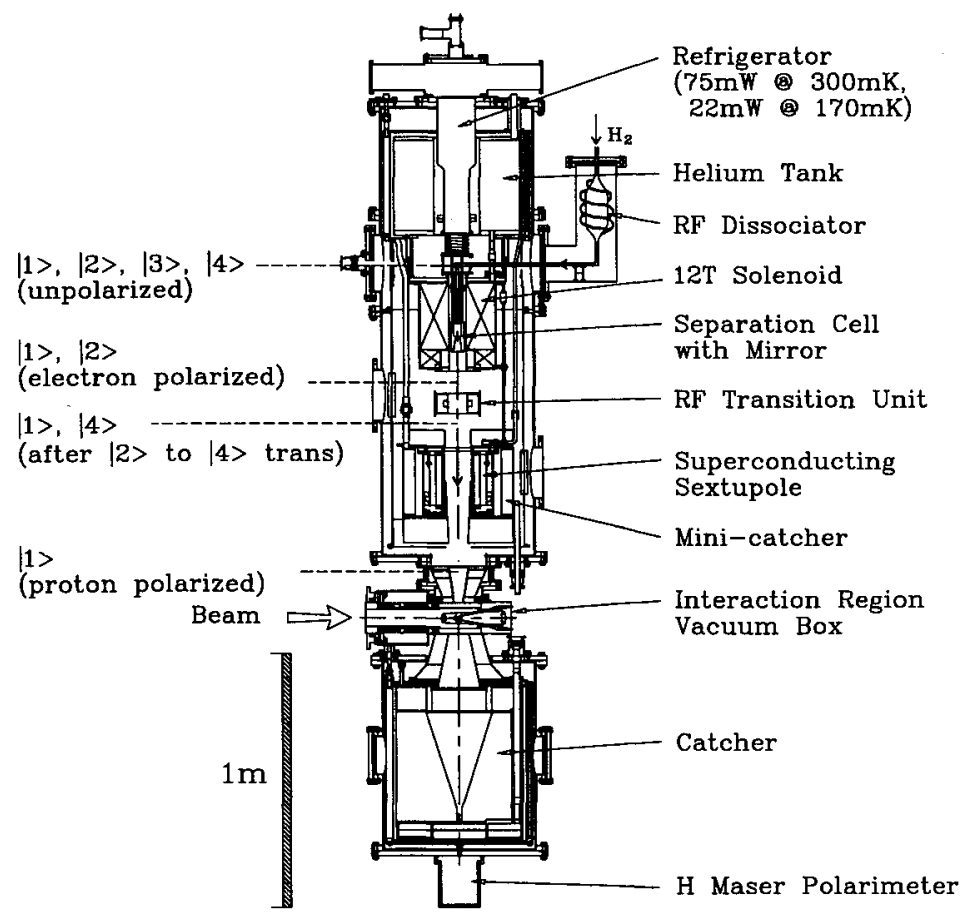

FIGURE 1. Layout of the Michigan ultra-cold Jet

atoms, the beam passes through the superconducting sextupole magnet. The sextupole focuses state $|1\rangle$ atoms into the interaction region and defocusses state $|4\rangle$ atoms, which are then cryopumped away. The proton-spin-polarized beam will then pass through the interaction region where it can collide with a proton beam in a high energy storage ring. The Jet beam is captured below by a huge cryopumping catcher [4] to keep the storage ring's vacuum uncontaminated. A maser polarimeter below the catcher monitors the Jet's proton polarization.

Most of the Michigan Jet parts have been fabricated, successfully tested, and used. This hardware includes a 12 Tesla superconducting solenoid with a very sharp gradient at its downstream end, a dilution refrigerator with a cooling power of about $22 \mathrm{~mW}$ at $170 \mathrm{mK}$, a $20 \mathrm{~cm}$ long superconducting sextupole magnet with a $3.1 \mathrm{kGauss}$ field at its iron poles' $10.5 \mathrm{~cm}$ diameter bore, the catcher with a measured cryocondensation pumping speed of $1.210^{7}$ liters $\mathrm{s}^{-1}$ [4], and a hydrogen maser polarimeter capable of monitoring the polarization with a precision of about $\pm 2 \%$ in a few minutes.

We studied a beam of polarized hydrogen atoms, which were focused by the superconducting sextupole into a compression tube detector that measured the polarized atoms' intensity. To avoid the superfluid ${ }^{4} \mathrm{He}$ film creeping out of the ultra-cold region, we installed three film burners above the mixing chamber, as shown in Fig. 2. Each film burner consists of a double-walled cell and a heater which is wound outside the cell. The superfluid ${ }^{4} \mathrm{He}$ film which creeps into the film burner cell is evaporated by the heater; the ${ }^{4} \mathrm{He}$ 
gas then recondences inside the the double-walled cell. By applying a higher heater voltage, we were able to build a thicker ${ }^{4} \mathrm{He}$ superfluid film, as shown in Fig. 3; this thicker film clearly increased the hydrogen beam intensity over some range, as shown in Fig. 3. The hydrogen intensity decreased when the film burner voltage was higher than $0.55 \mathrm{~V}$ since the heater power was then too high to recondense the ${ }^{4} \mathrm{He}$ gas. We concluded that the optimum film burner voltage was near $0.5 \mathrm{~V}$.

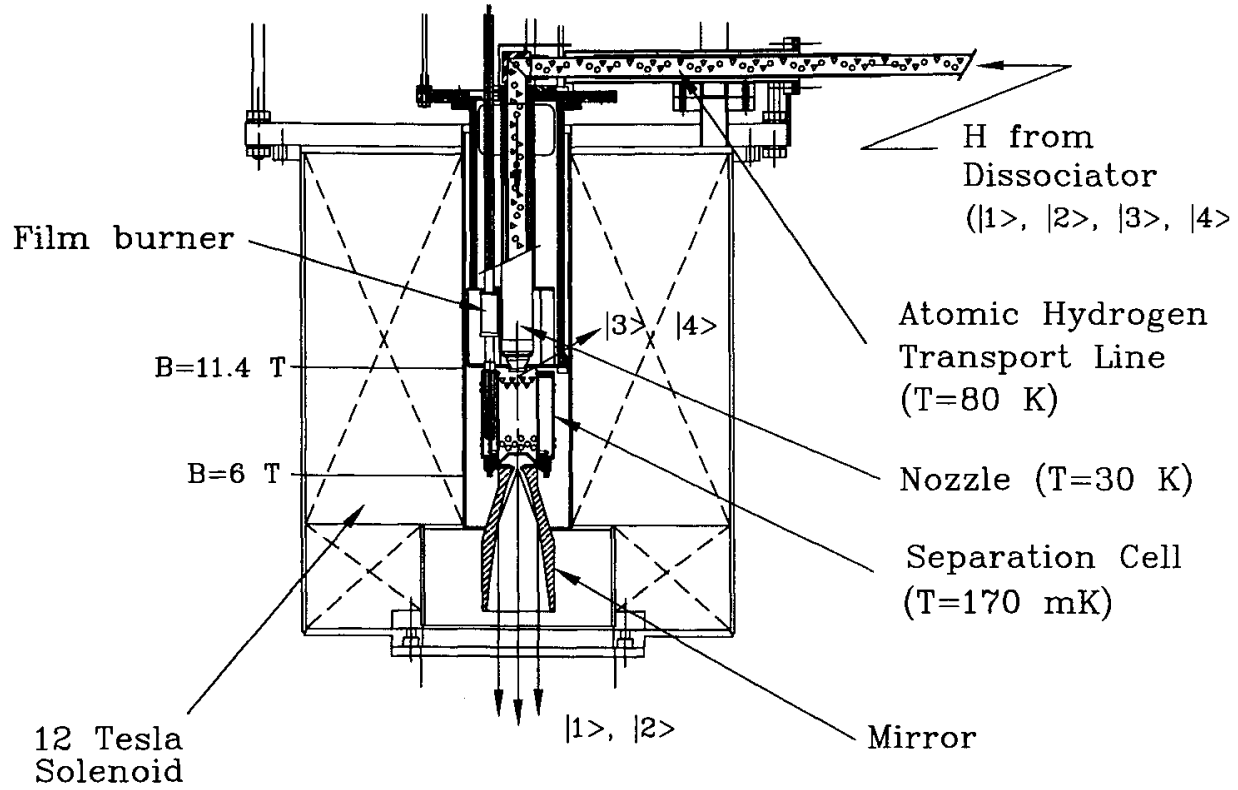

FIGURE 2. Details of the Michigan Jet's electron-spin separation region.

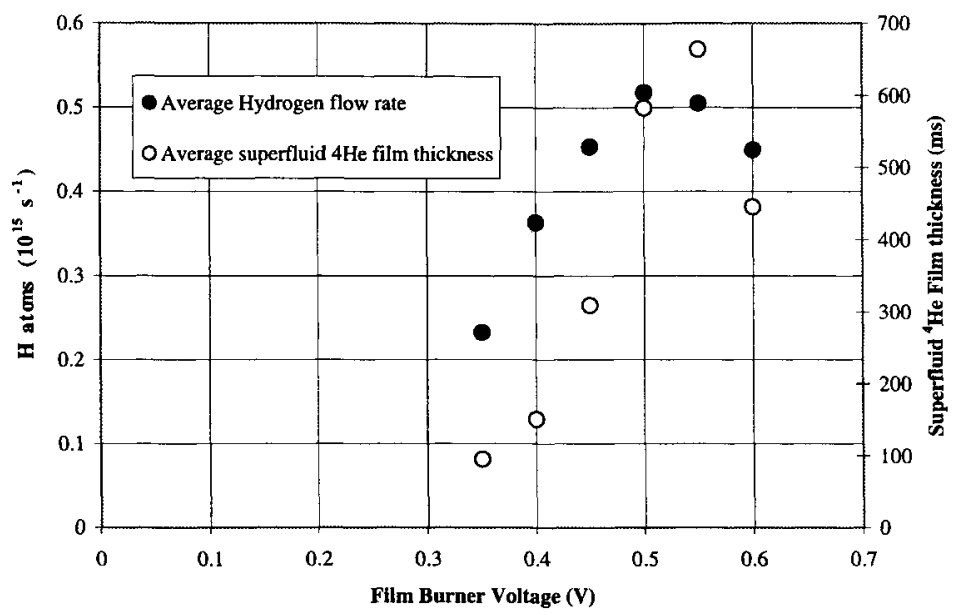

FIGURE 3. The observed hydrogen intensity entering the compression tube and superfluid ${ }^{4} \mathrm{He}$ film thickness as a function of the film burner voltage. 


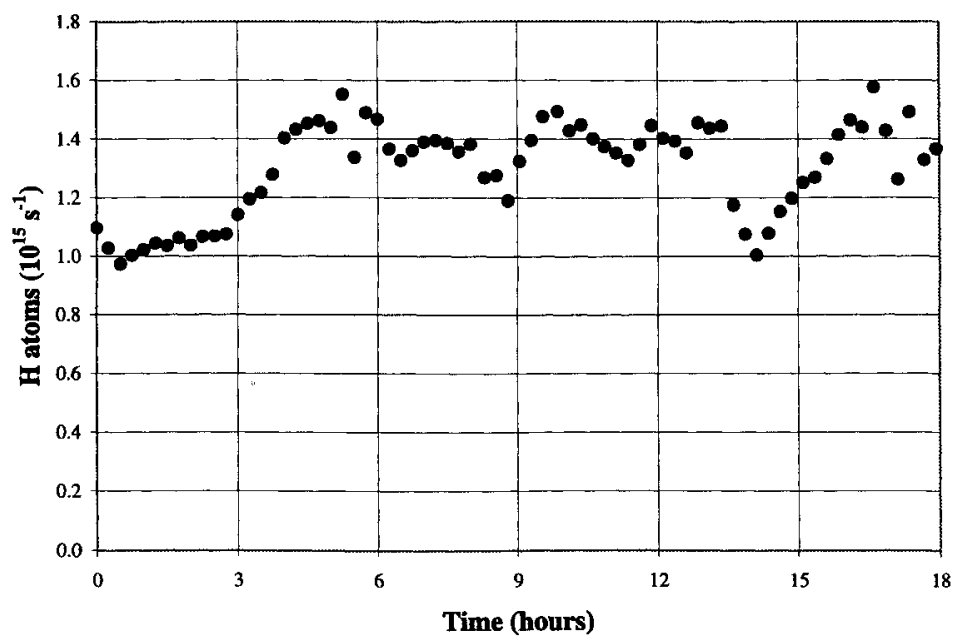

FIGURE 4. The observed hydrogen Jet intensity entering the compression tube during an 18-hour run.

By building a thick ${ }^{4} \mathrm{He}$ superfluid film, we were able to produce a high intensity spin-polarized hydrogen beam, which operated with good stability during an 18-hour run, until our liquid ${ }^{4} \mathrm{He}$ supply was depleted; the data is shown in Fig. 4. The average measured hydrogen intensity, into the $11 \mathrm{~mm}$ by $1.4 \mathrm{~mm}$ compression tube slot, was about $1.110^{15} \mathrm{H} \mathrm{s}^{-1}$. This intensity corresponds to a hydrogen Jet thickness of 0.8 $10^{12} \mathrm{H} \mathrm{cm}^{-2}$; the maximum fluctuation was about $\pm 20 \%$. The Jet's highest measured intensity was $2.210^{15} \mathrm{H} \mathrm{s}^{-1}$ [5]; this gave a Jet thickness of about $1.110^{12} \mathrm{H} \mathrm{cm}^{-2}$. We can heat the separation cell to about $40 \mathrm{~K}$ to remove the residual frozen $\mathrm{H}_{2}$ molecules; this usually takes about 2 hours, but it was not needed during this 18-hour run.

We thank D. Kleppner and T. Roser for their earlier help.

\section{REFERENCES}

1. B.B. Blinov et al., "Michigan Ultra-Cold Polarized Atomic Hydrogen Jet", in Proc. of 14th International Spin Physics symposium, Osaka 2000, edited by K. Hatanaka et al., American Institute of Physics, New York (2001), AIP Proceedings 570, pp.856-860.

2. V.G. Luppov et al., Phys. Rev. Lett., 71, 2405 (1993).

3. R.S. Raymond, "Development of a Large-bore Cryogenic 2-4 Transition Unit", in Proc. of International Workshop on Polarized Sources and Targets, edited by A. Gute et al., Erlangen, Germany (1999).

4. V.G Luppov et al., "Status of the Mark-II Polarized Hydrogen Jet Target", in Proc. of the 12th International Symposium on High-Energy Spin Physics, edited by C. de Jager et al., New York (1997), World Scientific, pp. 434-437.

5. V.G. Luppov, "Status of the Michigan Ultra-Cold Polarized Hydrogen Target," in Proc. of 9th International Workshop on Polarized Sources and Targets, edited by V.P. Derenchuk et al., Indiana (2001), World Scientific, pp. 32-36. 